\section{(6) OPEN ACCESS}

- Additional material is published online only. To view please visit the journal online (http://dx.doi.org/10.1136/ gutjnl-2013-304738).

For numbered affiliations see end of article.

\section{Correspondence to} Dr Kristian B Filion, Division of Clinical Epidemiology, McGill University, Lady Davis Research Institute, Jewish General Hospital, 3755 Cote Ste Catherine, Suite H4.16.1, Montreal, Quebec, Canada H3T 1E2; kristian.filion@mcgill.ca

Received 19 February 2013 Revised 24 May 2013 Accepted 3 June 2013 Published Online First 16 July 2013

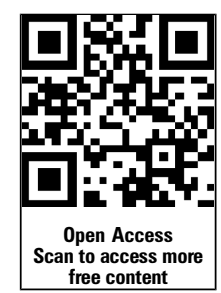

To cite: Filion $\mathrm{KB}$, Chateau D, Targownik LE, et al. Gut 2014;63: 552-558.

\title{
Proton pump inhibitors and the risk of hospitalisation for community-acquired pneumonia: replicated cohort studies with meta-analysis
}

\author{
Kristian B Filion, ${ }^{1}$ Dan Chateau, ${ }^{2}$ Laura E Targownik, ${ }^{3}$ Andrea Gershon, ${ }^{4}$ \\ Madeleine Durand, ${ }^{5}$ Hala Tamim, ${ }^{6}$ Gary F Teare, ${ }^{7}$ Pietro Ravani, ${ }^{8}$ Pierre Ernst, ${ }^{1}$ \\ Colin R Dormuth, ${ }^{9}$ the CNODES Investigators
}

\begin{abstract}
Objective Previous observational studies suggest that the use of proton pump inhibitors (PPIs) may increase the risk of hospitalisation for community-acquired pneumonia (HCAP). However, the potential presence of confounding and protopathic biases limits the conclusions that can be drawn from these studies. Our objective was, therefore, to examine the risk of HCAP with PPIs prescribed prophylactically in new users of non-steroidal anti-inflammatory drugs (NSAIDs).

Design We formed eight restricted cohorts of new users of NSAIDs, aged $\geq 40$ years, using a common protocol in eight databases (Alberta, Saskatchewan, Manitoba, Ontario, Quebec, Nova Scotia, US MarketScan and the UK's General Practice Research Database (GPRD)). This specific patient population was studied to minimise bias due to unmeasured confounders. Highdimensional propensity scores were used to estimate site-specific adjusted ORs (aORs) for HCAP at 6 months in PPI patients compared with unexposed patients.

Fixed-effects meta-analytic models were used to estimate overall effects across databases.
\end{abstract}

Results Of the 4238504 new users of NSAIDs, 2.3\% also started a PPI. The cumulative 6-month incidence of HCAP was $0.17 \%$ among patients prescribed PPIs and $0.12 \%$ in unexposed patients. After adjustment, PPIs were not associated with an increased risk of HCAP $(\mathrm{aOR}=1.05 ; 95 \% \mathrm{Cl} 0.89$ to 1.25$)$. Histamine-2 receptor antagonists yielded similar results $(\mathrm{aOR}=0.95,95 \%$ $\mathrm{Cl} 0.75$ to 1.21$)$.

Conclusions Our study does not support the proposition of a pharmacological effect of gastric acid suppressors on the risk of HCAP.

\section{INTRODUCTION}

Overutilisation of proton pump inhibitors (PPIs) and their potential health risks are attracting increasing attention. ${ }^{12}$ One of the suspected health concerns associated with their use is a possible increase in the risk of pneumonia. ${ }^{3}$ The proposed mechanism behind this potential effect is bacterial overgrowth of the stomach and oesophagus increasing the risk of bacterial aspiration. Although evidence from previous observational studies support the existence of an association between the use of PPIs and the risk of community-acquired pneumonia, ${ }^{3}$ these studies had important limitations. These limitations include confounding due to gastroesophageal reflux disease

\section{Significance of this study}

What is already known on this subject?

- Previous observational studies and their meta-analysis have found that proton pump inhibitors are associated with an increased risk of community-acquired pneumonia.

- Potential confounding by gastroesophageal reflux disease and protopathic bias limit the conclusions that can be drawn from these studies.

- Proton pump inhibitors are also prescribed prophylactically with non-steroidal anti-inflammatory drugs, and the study of this population may overcome the limitations of previous studies examining this association.

What are the new findings?

- Proton pump inhibitors are not associated with an increased risk of hospitalisation for community-acquired pneumonia (HCAP) (adjusted $\mathrm{OR}=1.05 ; 95 \% \mathrm{Cl} 0.89$ to 1.25 ).

- There is also no association between histamine-2 receptor antagonists and the risk of HCAP (adjusted OR=0.95, 95\% Cl 0.75 to 1.21 ), suggesting a lack of dose-response relationship between potency of gastric acid suppression and the risk of HCAP.

How might it impact on clinical practice in the foreseeable future?

- Our results suggest that concerns regarding this association should not influence prescribing of gastric acid-suppressing medications.

(GERD), a potentially independent risk factor for pneumonia, ${ }^{4} 5$ and with a sharp increase in risk observed shortly after PPI initiation, ${ }^{367}$ the likely presence of protopathic bias.

Although PPIs are most commonly prescribed for the treatment of symptoms of GERD, they may also be prescribed concomitantly with non-steroidal anti-inflammatory drugs (NSAIDs) to prevent ulcer formation and dyspepsia. ${ }^{8-10}$ As patients who are prescribed PPIs for this indication are less likely to have GERD, an analysis restricted to this specific 
cohort may help isolate the independent contribution of PPI exposure to the risk of hospitalisation for community-acquired pneumonia (HCAP) by minimising bias from unmeasured confounders. We therefore examined the risk of HCAP with PPIs prescribed prophylactically in a cohort of new users of NSAIDs who were not previously exposed to gastric acid-suppressing medications. We also examined the association between HCAP and histamine-2 receptor antagonists (H2RAs), a less potent class of gastroprotective agents, to investigate the effect of gastric acid suppression potency on the risk of incident HCAP. Our a priori hypothesis was that use of PPIs and H2RAs would result in an increased risk of incident HCAP relative to non-use.

\section{METHODS}

\section{Study population}

We applied a common protocol to databases from eight jurisdictions (Alberta, Saskatchewan, Manitoba, Ontario, Quebec, Nova Scotia, US MarketScan, and the UK's General Practice Research Database (GPRD)) as part of the Canadian Network for Observational Drug Effect Studies (CNODES). ${ }^{11}$ Within each jurisdiction, we conducted a retrospective cohort study of all individuals aged $\geq 40$ years who were prescribed an oral NSAID (WHO Anatomical Therapeutic Chemical (ATC) Code M01A) for the first time between 1 January 1997 and 31 March 2010. Patients were permitted to enter the study multiple times provided that all inclusion criteria were met. ${ }^{12}$ The six Canadian databases encompass $>90 \%$ of the entire population of their respective provinces, with excluded individuals being those whose drug plan coverage is funded federally (eg, members of the armed forces, inmates of federal penitentiaries). The GPRD includes a representative sample of the UK's population, and MarketScan contains data for those covered by large US employer health insurance plans. Analyses in Alberta, Nova Scotia, Ontario and Quebec were limited to those aged $\geq 66$ years as prescription drug data were not available for younger patients. Analyses conducted using MarketScan were limited to those aged 40-65 years, as complete data capture was not possible in older patients due to their eligibility for Medicare. Due to data availability, analyses conducted using Quebec data were restricted to a $10 \%$ random sample of patients covered by the provincial drug plan. In all analyses, the study population was restricted to those receiving an NSAID prescription of $\geq 28$ days duration as patients receiving short-duration prescriptions are unlikely to be prescribed a PPI for prophylactic reasons. For prescriptions missing duration, duration was estimated by multiplying the dosage by the quantity dispensed, and dividing by WHO-defined daily dosage. Cohort entry was defined by the date of a patient's NSAID prescription.

We excluded all patients who met any of the following criteria: (1) aged $<40$ years at cohort entry due to the low incidence of HCAP in this population; (2) received a prescription for a PPI, a H2RA, or a NSAID (any route of administration) in the 6 months prior to cohort entry; (3) had an HCAP (ICD-9-CM code (in any field): 480.x-487.x; ICD-10-CA code: J10.0 - J18.9) or an extended emergency room visit for community-acquired pneumonia (defined as the presence of emergency room billing codes for pneumonia on two consecutive days) in the year prior to cohort entry in databases in which emergency room data were available; (4) hospitalised at the time of cohort entry; (5) received a prescription for medications used for the treatment of tuberculosis (ATC Code J04A) in databases where these data were available; (6) had a history of cancer (other than non-melanoma skin cancer) in the year prior to cohort entry as cancer and its treatments may increase the risk of pneumonia; (7) hospitalised $\geq 3$ days within the 30 days before cohort entry to ensure that all events were community-acquired and (8) had $<1$ year of continuous observation time in the database prior to cohort entry to ensure complete assessment of relevant medical history.

Patients meeting the study inclusion criteria were followed until a first-ever HCAP (defined below), death from any cause, admission for a hospitalisation with a length of stay $>3$ days, departure from the database, end of follow-up (180 days), or end of the study period (30 September 2011), whichever came first. Research ethics approval was obtained at all sites.

\section{Exposure definition}

We obtained all information for medications dispensed by pharmacies for all patients, except for the GPRD, which collects prescriptions entered electronically. Exposure was defined using three mutually exclusive categories. Patients receiving a prescription for any of the following oral PPIs (alone or in combination therapy) on the same day as their NSAID prescription (cohort entry) were considered exposed to PPIs: esomeprazole, omeprazole, pantoprazole, lansoprazole and rabeprazole. The second category included patients prescribed any of the following oral H2RAs on the same day as their NSAID prescription (cohort entry): cimetidine, ranitidine, famotidine, nisatidine, niperotidine, roxatidine, ranitidine bismuth citrate, lafutidine, cimetidine combinations and famotidine combinations. The third category, which served as the reference category, consisted of those not prescribed a PPI or H2RA on the same day as the NSAID prescription defining cohort entry. Patients prescribed both an H2RA and PPI on the day of cohort entry were excluded.

In our primary analysis, exposure was defined using an intention-to-treat approach. In sensitivity analyses, we examined the effect of misclassification of exposure by excluding initially unexposed patients prescribed a PPI or H2RA during follow-up.

\section{Outcome definition}

The primary outcome was incident HCAP during the 6 months following initiation of NSAID therapy. HCAP was defined using a validated algorithm based on ICD-10 discharge codes. ${ }^{13}$ In a previous validation study conducted using data from Alberta, this definition was associated with positive and negative predictive values of $96 \%$ and $98 \%$ compared with data extracted from patients' charts and $71 \%$ and $84 \%$ compared with chest X-ray reports. The definition was restricted to codes identified as the most responsible diagnosis that did not also appear as a code indicating that the condition arose during hospitalisation. In Quebec, the event definition also included extended emergency room visits for pneumonia. Sensitivity analyses were performed where these visits were excluded. The date of HCAP was defined as the date of the hospital admission.

\section{Data analysis}

We used multiple logistic regression to estimate adjusted ORs (aOR) and corresponding 95\% CIs. As the proportion of patients with HCAP during the 6-month follow-up period is small, the estimated ORs approximate the cumulative incidence ratios. ${ }^{14}$ To further minimise bias from confounding by indication and other factors, we constructed high-dimensional propensity scores. ${ }^{15}$ The following variables were forced into the propensity score model: index year, income category, hospitalisation in the previous year, use of $>4$ medications in the previous year, $>4$ physician visits in the previous year, asthma, chronic obstructive pulmonary disease, bronchiectasis, diabetes 
and use of immunosuppressive agents, inhaled bronchodilators, inhaled corticosteroids, non-topical antibiotics, non-topical corticosteroids and, where data were available, use of influenza or pneumococcal vaccines. Excessive alcohol use, body mass index (BMI) and smoking were also forced into the propensity score model fit in the GPRD. In addition to these prespecified potential confounders, up to 500 additional confounders were empirically selected for inclusion in the propensity score models. Comorbidities were assessed using discharge diagnoses in the year before cohort entry, and medications were assessed using prescriptions in the year before cohort entry.

Following the estimation of the high-dimensional propensity score for each patient using a logistic model, patients were categorised into propensity score deciles, which were included as a categorical variable in our final logistic model to estimate the independent effect of PPIs on the risk of HCAP. We also adjusted for age, sex, previous non-hospitalised pneumonia, and prescription of PPIs, H2RAs and NSAIDs in the 7-12 months before cohort entry (prescriptions in the 6 months before cohort entry resulted in exclusion) in our outcome model. In order to account for the possibility of multiple cohort entry dates for each patient, generalised estimating equations were used. Analyses were then repeated to estimate the effect of H2RAs on the risk of HCAP. PPIs and H2RAs were examined separately to allow for the calculation of separate highdimensional propensity scores for each exposure.

We conducted additional sensitivity analyses to verify the robustness of our results. First, analyses were restricted to one randomly selected observation per patient to reduce the influence of intrasubject correlation. Second, we excluded patients who received a prescription for a PPI, H2RA, or NSAID in the previous 12 months rather than in the prior 6 months to further increase the probability that patients with GERD were excluded. Finally, we repeated analyses excluding 'crossovers': unexposed patients who received a PPI or H2RA during follow-up, PPI users also exposed to H2RAs during follow-up, and H2RA users also exposed to PPIs during follow-up. All site-specific analyses were conducted using SAS V.9.2 (The SAS Institute, Cary, North Carolina, USA).

Site-specific estimates were then pooled using fixed-effect meta-analytic models with inverse variance weighting. ${ }^{16}$ The amount of between-site heterogeneity was estimated by the $\mathrm{I}^{2}$ statistic. $^{17}$ In sensitivity analyses, data were pooled using random-effects models. In additional sensitivity analyses, data from Nova Scotia were excluded as the Nova Scotia Seniors' Pharmacare program required specific criteria to be followed prior to PPI reimbursement until 11 January 2008. ${ }^{18}$ All meta-analyses were conducted using Stata V.11.2 (StataCorp LP, College Station, Texas, USA).

\section{RESULTS}

\section{Study population}

When data were pooled across all sites, our PPI analyses included 96870 exposed and 4141634 unexposed patients, and our H2RA analyses included 47344 exposed and 4342733 unexposed patients. All sites contributed data to the PPI analysis except Saskatchewan, where PPI data were incomplete because PPIs are listed in the formulary with restricted coverage and, therefore, require approval under the Exception Drug Status program in order to be captured in the database.

Compared with unexposed patients, those prescribed PPIs were more likely to be older, female and had a higher burden of comorbidities (table 1). In the GPRD, where additional information was available, patients prescribed PPIs were also more likely to have a history of excessive alcohol use $(4.1 \%$ vs $2.8 \%)$ and a history of smoking $(53.4 \%$ vs $49.8 \%$ among those with smoking data) but a similar proportion of patients with a BMI $\geq 30 \mathrm{~kg} / \mathrm{m}^{2}$ (both $29.7 \%$ among those with BMI data). Not surprisingly, in all databases, exposed patients were more likely to have been prescribed a PPI in the 7-12 months before cohort entry. Patients prescribed PPIs were also more likely to have been prescribed vaccinations for influenza and pneumonia. Similar patterns in baseline characteristics were observed when comparing patients prescribed H2RAs to unexposed patients (see online supplementary appendix 1). Additionally, similar distributions were observed when inclusion was restricted to one, randomly selected observation per patient (see online supplementary appendix 2).

\section{Primary analysis}

The cumulative 6 -month incidence of HCAP was $0.17 \%$ among patients prescribed PPIs and $0.12 \%$ in unexposed patients. When data were pooled across all databases, PPIs were not associated with the incidence of HCAP $(\mathrm{aOR}=1.05 ; 95 \%$ CI 0.89 to $1.25 ; \mathrm{I}^{2}=0 \%$ ) (figure 1). All site-specific aORs were between 0.93 and 1.21 except for that of Nova Scotia $(\mathrm{aOR}=3.73 ; 95 \%$ CI 1.12 to 12.4 ). When analyses were repeated excluding Nova Scotia, results were consistent with those of our primary analysis ( $\mathrm{aOR}=1.03,95 \%$ CI 0.87 to $\left.1.22 \mathrm{I}^{2}=0 \%\right)$.

Analyses of the association between H2RAs and HCAP yielded similar results (6-month cumulative incidence $=0.16 \%$ in the H2RA group vs $0.12 \%$ in the unexposed group; $\mathrm{aOR}=0.95$, $95 \%$ CI to 0.75 to $1.21 ; \mathrm{I}^{2}=0 \%$ ) (figure 2 ). Quebec did not contribute to this analysis as there were no HCAPs that occurred in H2RA users in their 10\% data sample.

\section{Sensitivity analyses}

We conducted multiple sensitivity analyses, including analyses restricted to a single, random, observation per patient analyses, that excluded those who received a prescription for a PPI, H2RA, or NSAID in the 12 months before cohort entry, and analyses that excluded crossovers. These sensitivity analyses produced results that were consistent with those of our primary analyses (table 2, see online supplementary appendices 3-8). Additionally, similar results were obtained in sensitivity analyses involving the use of random-effects meta-analyses (data not shown).

\section{DISCUSSION}

Among new users of NSAIDs, we found that PPIs were not associated with the risk of HCAP. Similar results were obtained across all sites except Nova Scotia, where formulary restrictions introduced strong confounding by indication. We also observed no association between H2RAs and HCAP.

The potential association between use of gastric acidsuppressing medications and pneumonia has been examined

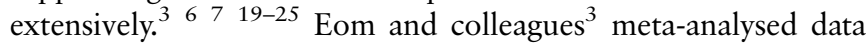
across eight observational studies and found that PPI use was associated with a $27 \%$ increased risk of either hospital-acquired or community-acquired pneumonia $(\mathrm{aOR}=1.27,95 \% \mathrm{CI} 1.11$ to 1.46). This increased risk was predominantly due to an increased risk of community-acquired pneumonia (five studies: $\mathrm{aOR}=1.34$, 95\% CI 1.14 to 1.57 ). Importantly, a strong temporal relationship was observed, with a duration of exposure $<7$ days being associated with a three-fold increase in the risk of pneumonia $(\mathrm{aOR}=3.95,95 \%$ CI 2.86 to 5.45$)$. These apparent short-term effects are further illustrated in the nested case-control study by Sarkar and colleagues, ${ }^{20}$ who found a null association overall but 
Table 1 Demographic and clinical characteristics by exposure status at cohort entry in a restricted cohort of new users of NSAIDs

\begin{tabular}{|c|c|c|}
\hline & \multicolumn{2}{|l|}{ PPI analysis } \\
\hline & PPI exposed & Unexposed* \\
\hline Total number of patients & 96870 & 4141634 \\
\hline \multicolumn{3}{|l|}{ Database (\%) } \\
\hline Alberta & 6.1 & 4.3 \\
\hline Saskatchewan & 0 & 0 \\
\hline Manitoba & 3.0 & 5.5 \\
\hline Ontario & 34.2 & 22.8 \\
\hline Quebec & 1.0 & 0.7 \\
\hline Nova Scotia & 0.5 & 1.0 \\
\hline MarketScan & 25.9 & 52.1 \\
\hline GPRD & 29.4 & 13.7 \\
\hline \multicolumn{3}{|l|}{ Age $(\%)$ (years) $†$} \\
\hline $40-54$ & 24.9 & 41.6 \\
\hline $55-64$ & 20.0 & 23.9 \\
\hline $65-74$ & 30.5 & 19.5 \\
\hline $75-84$ & 19.8 & 12.2 \\
\hline $85+$ & 4.9 & 2.8 \\
\hline Women (\%) & 62.8 & 58.3 \\
\hline \multicolumn{3}{|l|}{ Year of cohort entry (\%) } \\
\hline 1997 & 0.2 & 1.3 \\
\hline 1998 & 0.5 & 2.3 \\
\hline 1999 & 0.6 & 2.9 \\
\hline 2000 & 1.4 & 4.4 \\
\hline 2001 & 2.2 & 4.6 \\
\hline 2002 & 3.0 & 4.6 \\
\hline 2003 & 4.4 & 4.8 \\
\hline 2004 & 5.7 & 4.6 \\
\hline 2005 & 7.2 & 3.7 \\
\hline 2006 & 9.0 & 3.7 \\
\hline 2007 & 17.1 & 18.5 \\
\hline 2008 & 21.0 & 19.0 \\
\hline 2009 & 20.9 & 18.0 \\
\hline 2010 & 6.6 & 7.6 \\
\hline 2011 & 0.0 & 0.0 \\
\hline Nursing home $(\%) \ddagger$ & 0.7 & 0.8 \\
\hline Urban (\%)§ & 85.5 & 78.8 \\
\hline \multicolumn{3}{|l|}{ Comorbidities (\%)ף } \\
\hline Asthma & 2.6 & 1.8 \\
\hline Bronchiectasis & 0.1 & 0.1 \\
\hline COPD & 3.1 & 2.3 \\
\hline Diabetes & 9.9 & 9.7 \\
\hline Non-hospitalised pneumonia & 2.2 & 2.2 \\
\hline \multicolumn{3}{|c|}{ Hospitalisations in the year preceding cohort entry (\%) } \\
\hline None & 91.7 & 93.2 \\
\hline One & 6.8 & 5.6 \\
\hline Two & 1.1 & 0.9 \\
\hline Three & 0.3 & 0.2 \\
\hline Four or more & 0.1 & 0.1 \\
\hline \multicolumn{3}{|c|}{ Prescriptions in the year preceding cohort entry (\%) } \\
\hline None & 4.0 & 5.2 \\
\hline One & 6.1 & 8.2 \\
\hline Two & 7.4 & 9.7 \\
\hline Three & 8.4 & 10.2 \\
\hline Four or more & 74.1 & 66.7 \\
\hline \multicolumn{3}{|l|}{ Medications (\%)ף } \\
\hline $\mathrm{PPI}^{* *}$ & 25.3 & 3.0 \\
\hline $\mathrm{H}_{2} \mathrm{RA}^{* *}$ & 2.6 & 1.6 \\
\hline NSAIDs** & 30.7 & 28.5 \\
\hline
\end{tabular}

Table 1 Continued

\begin{tabular}{llc}
\hline & \multicolumn{2}{l}{ PPI analysis } \\
\cline { 2 - 3 } & PPI exposed & Unexposed* $^{*}$ \\
\hline Immunosuppressive agents & 1.3 & 1.1 \\
Influenza vaccinet† & 20.9 & 5.6 \\
Inhaled bronchodilators & 11.4 & 8.4 \\
Inhaled corticosteroids & 11.2 & 13.6 \\
Pneumococcal vaccinet† & 11.9 & 2.4 \\
Non-topical antibiotics & 43.4 & 45.0 \\
Non-topical corticosteroids & 13.5 & 16.8
\end{tabular}

* Saskatchewan did not contribute to the PPI analysis. In addition, small differences in the unexposed groups exist in the PPI and H2RA analyses because of trimming during the estimation of high-dimensional propensity scores.

tAlberta, Ontario, Quebec and Nova Scotia only contributed data for those $>66$ years of age whereas MarketScan only contributed data for those aged 40-65 years.

$\ddagger$ The proportion of study participants living in nursing homes was only reported by Manitoba and Ontario.

$\S$ Data regarding urban/rural location were only available in Alberta, Manitoba, Nova Scotia and Ontario.

ПComorbidities and medication use are defined using data in the year prior to and including the date of cohort entry.

**Denotes use in the 7-12 months before cohort entry. Use in the 6 months before cohort entry resulted in exclusion.

††Vaccination data were only available for the GPRD, Manitoba, MarketScan and Nova Scotia.

COPD, chronic obstructive pulmonary disease; GPRD, General Practice Research Database; H2RA, histamine-2 receptor antagonist; NSAIDs, non-steroidal anti-inflammatory drugs; PPI, proton pump inhibitor.

a substantially increased risk of community-acquired pneumonia in those who initiated PPI therapy in the 2 days before the index date $(\mathrm{aOR}=6.5,95 \%$ CI 3.95 to 10.80$)$. Such a temporally close increased risk, before PPIs achieve full effect, is suggestive of protopathic bias due to early signs of pneumonia, including nonspecific chest symptoms and discomfort, being misdiagnosed as GERD. These studies were also likely affected by confounding by GERD, which has been associated with an increased risk of respiratory symptoms, and may promote the translocation of gastric contents into the respiratory tract. ${ }^{26} 27$ The role of confounding in the purported PPI-pneumonia association has recently been examined by Jena and colleagues, who found that PPI users also had increased risk of other medical conditions thought to be unrelated to PPI use. ${ }^{28}$ Our null results, both from our primary analyses and in sensitivity analyses that excluded all patients who received a prescription for a PPI, H2RA, or NSAID in the year before cohort entry, suggest that the PPI-pneumonia association found in previous observational studies ${ }^{6719-23}$ and published meta-analyses ${ }^{324}$ was likely due to the presence of confounding and/or protopathic bias; these limitations were minimised in the present study by our use of a restricted cohort and an intention-to-treat approach.

In addition to these previous observational studies, the association between PPIs and community-acquired pneumonia was examined in a retrospective analysis of 31 trials of esomeprazole. $^{29}$ The rate of pneumonia was similar in those receiving this PPI and those treated with placebo or non-PPI comparator $(\mathrm{OR}=0.94,99 \% \mathrm{CI} 0.29$ to 3.07$)$. However, the generalisability of these trial data to a real-world setting is unclear. Furthermore, despite the large sample size $(n=16000)$, the wide $99 \%$ CI suggests that the analysis was underpowered to identify clinically important effects.

Our study has a number of strengths. First, although the use of PPIs specifically to prevent NSAID-related gastrointestinal complications is relatively uncommon, analysis of data from seven sites resulted in a sufficiently large source population to 
Figure 1 Forest plot of the association between the use of proton pump inhibitors (PPIs) and the 6-month cumulative incidence of hospitalisation for community-acquired pneumonia in a restricted cohort of new users of non-steroidal anti-inflammatory drugs (NSAIDs). Analyses were adjusted for age, sex, previous non-hospitalised pneumonia, prescription of PPIs, histamine-2 receptor antagonists and NSAIDs in the 7-12 months prior to cohort entry, and high-dimensional propensity score decile. GPRD, General Practice Research Database.

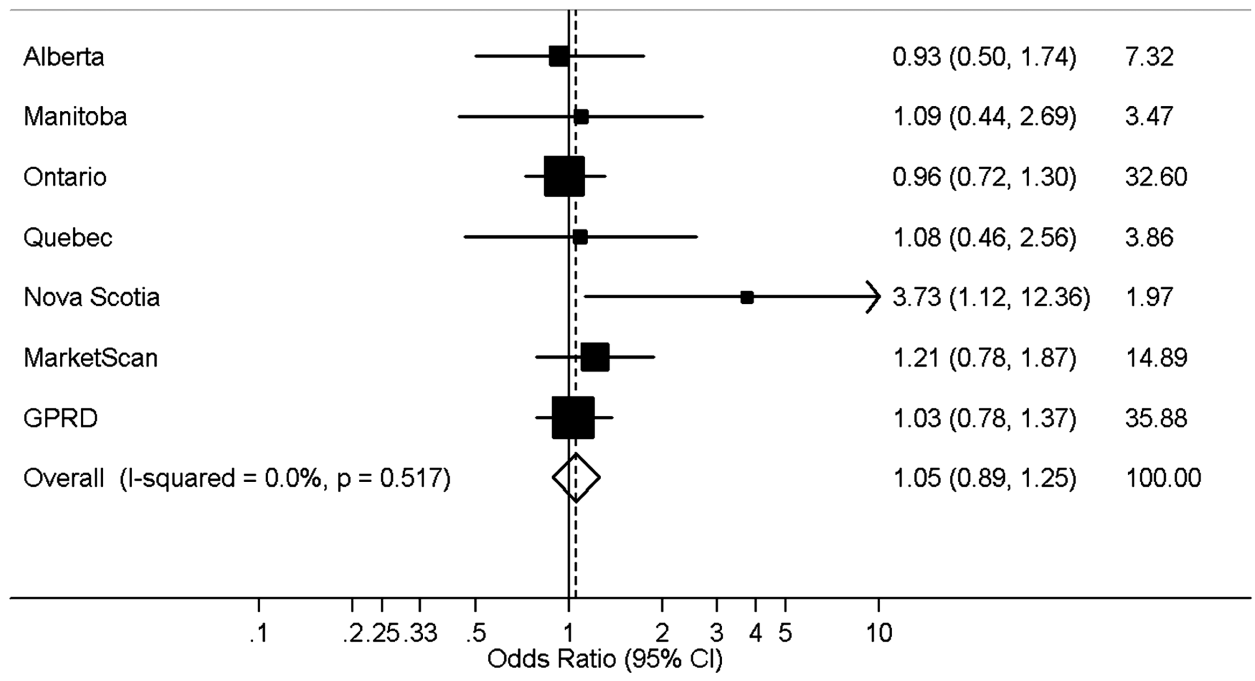

examine this association using a restricted cohort of new NSAID users, chosen to avoid confounding by indication and protopathic bias. Second, protection against confounding was also provided by our use of high-dimensional propensity scores. Third, our use of a distributed-protocol approach decreased the heterogeneity typically present in meta-analyses of observational studies while respecting legal and ethical privacy requirements. ${ }^{30}$ Fourth, while respiratory illnesses, such as pneumonia, chronic obstructive pulmonary disorder and acute asthma exacerbations may be misdiagnosed as one or the other, we used a validated HCAP definition, ${ }^{13}$ minimising potential misclassification. This study involved databases that have been used extensively for pharmacoepidemiologic investigations, and previous studies indicate that these data are valid. ${ }^{32-34}$ Finally, our use of a new user design avoided the underestimation of effects that frequently occurs with the study of prevalent users. ${ }^{12}$
Our study also has some potential limitations. First, at least two forms of H2RAs are available without a prescription in Canada (ranitidine $75 \mathrm{mg} / 150 \mathrm{mg}$ and famotidine $10 \mathrm{mg}$ / $20 \mathrm{mg}$ ), and some PPIs are available over the counter in the USA and the UK. Unfortunately, over-the-counter medication use is not captured by included databases. If there is a true increased risk of HCAP due to the use of 'gastroprotective agents', the exclusion of over-the-counter gastric acidsuppressing medications may bias the results towards the null. Second, we used an intention-to-treat approach, which mirrors that of a randomised controlled trial. This analytical approach may result in some exposure misclassification as some unexposed patients may have initiated therapy during follow-up and some exposed patients did not receive additional prescriptions during follow-up, resulting in a potential bias towards the null. However, sensitivity analyses that excluded unexposed patients

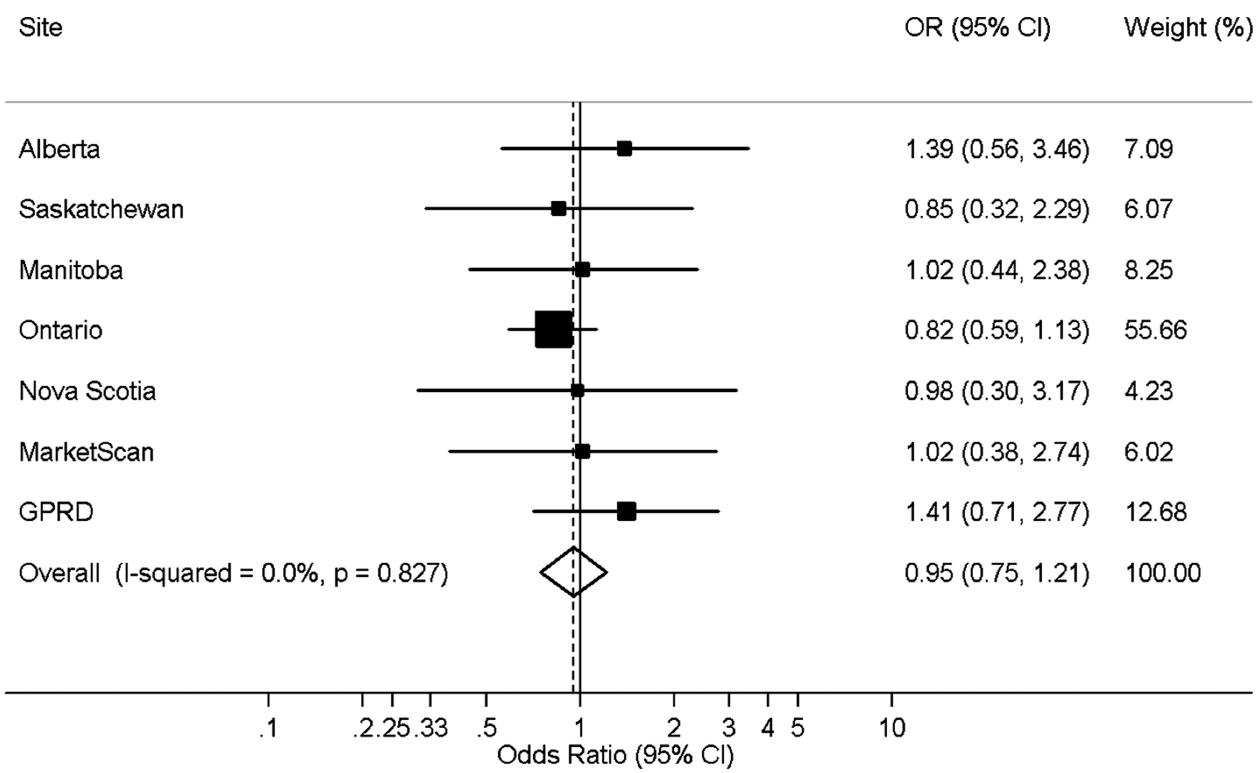

Figure 2 Forest plot of the association between the use of histamine-2 receptor antagonists and the 6-month cumulative incidence of hospitalisation for community-acquired pneumonia in a restricted cohort of new users of non-steroidal anti-inflammatory drugs (NSAIDs). Analyses were adjusted for age, sex, previous non-hospitalised pneumonia, prescription of proton pump inhibitors, histamine-2 receptor antagonists and NSAIDs in the 7-12 months prior to cohort entry, and high-dimensional propensity score decile. GPRD, General Practice Research Database. 
Table 2 Summary of analyses examining association between the use of gastric acid suppressing medications and the 6-month cumulative incidence of hospitalisation for community-acquired pneumonia in a restricted cohort of NSAIDs

\begin{tabular}{|c|c|c|}
\hline Exposure & Analysis & OR $(95 \% \mathrm{CI})$ \\
\hline \multirow[t]{4}{*}{ PPI } & Primary analysis & $1.05(0.89$ to 1.25$)$ \\
\hline & One random observation per patient & $1.08(0.90$ to 1.31$)$ \\
\hline & $\begin{array}{l}\text { Excluding patients prescribed a PPI, H2RA, or } \\
\text { NSAID in the year prior to cohort entry* }\end{array}$ & 1.08 (0.88 to 1.32$)$ \\
\hline & Excluding crossovers & $1.06(0.89$ to 1.27$)$ \\
\hline \multirow[t]{4}{*}{ H2RA } & Primary analysis & $0.95(0.75$ to 1.21$)$ \\
\hline & One random observation per patient & $0.97(0.74$ to 1.26$)$ \\
\hline & $\begin{array}{l}\text { Excluding patients prescribed a PPI, H2RA, or } \\
\text { NSAID in the year prior to cohort entry* }\end{array}$ & $1.06(0.78$ to 1.45$)$ \\
\hline & Excluding crossovers & $0.92(0.72$ to 1.18$)$ \\
\hline
\end{tabular}

who initiated PPI therapy during follow-up produced results that were consistent with those of our primary analysis. With exposure defined using written prescriptions (in the GPRD) and medication dispensations (the other seven sites), exposure misclassification due to poor patient adherence is also possible. Third, with the use of a restricted cohort, the generalisability of our results is unclear. For example, it is possible that the antiinflammatory properties of NSAIDs interfere with the harmful side effects of PPIs found in other studies. Fourth, data regarding the indication for acid suppression (ie, treatment of GERD, gastroprotection) were not available. Consequently, we were unable to use indication to further restrict our study cohort. Nonetheless, with inclusion restricted to those receiving an NSAID prescription with no prescriptions for PPIs or H2RAs in the prior 6 months (12 months in sensitivity analyses), the vast majority of PPI and H2RA prescriptions were likely for gastroprotection. Finally, while there is some evidence that coding practices for HCAP may have changed over time, ${ }^{35}$ it is unlikely that these changes were differential across exposure groups.

\section{CONCLUSIONS}

Our common protocol analysis does not support the hypothesis of an independent association between gastric acid-suppressing medications and the risk of HCAP. The positive results of previous studies examining this association are likely due to confounding and protopathic bias, which have been minimised in the present study through the use of a restricted cohort approach. Our results call a causal PPI-HCAP association into question and suggest that concerns regarding this association should not influence prescribing of gastric acid-suppressing medications.

\footnotetext{
Author affiliations

${ }^{1}$ Center for Clinical Epidemiology, Lady Davis Research Institute, Jewish General Hospital, McGill University, Montreal, Quebec, Canada

${ }^{2}$ Manitoba Centre for Health Policy, Department of Community Health Sciences, University of Manitoba, Winnipeg, Manitoba, Canada

${ }^{3}$ Section of Gastroenterology, Division of Internal Medicine, University of Manitoba, Winnipeg, Manitoba, Canada

${ }^{4}$ Department of Medicine, Sunnybrook Health Sciences Centre and Institute for Clinical Evaluative Sciences, Toronto, Ontario, Canada

${ }^{5}$ Internal Medicine Service, Centre Hospitalier Universitaire de Montréal (CHUM) and Pharmacoepidemiology and Pharmacoeconomy Research Unit of the CHUM, Montreal, Quebec, Canada
}

${ }^{6}$ School of Kinesiology and Health Science, York University, Toronto, Ontario, Canada

${ }^{7}$ Health Quality Council, Saskatoon, Saskatchewan, Canada

${ }^{8}$ Department of Medicine, University of Calgary, Calgary, Alberta, Canada ${ }^{9}$ Department of Anesthesiology, Pharmacology \& Therapeutics, University of British Columbia, Vancouver, British Columbia, Canada

Acknowledgements We thank Gregory Alan Carney BSc, Rick Chin MSC, Matthew Dahl BSc, Sophie Dell'Aniello MSc, Steve Doucette MSc, Thierry Ducruet MSc, Wenbin Li MSc, Yan Wang MSc and C Fangyun Wu for their programming support. We also acknowledge the contributions of Yola Moride, PhD at the Quebec site and those of Corine Mizrahi and Melissa Dahan at the Coordinating Center. We would like to acknowledge the important contributions of the CNODES collaborators and assistants at each site. This study was made possible through data sharing agreements between CNODES member research centres and the respective provincial governments of Alberta, British Columbia, Manitoba (HIPC \#: 2010/2011-66; HREB \#: H2011:104), Nova Scotia, Ontario, Quebec and Saskatchewan. The opinions, results and conclusions reported in this paper are those of the authors. No endorsement by the provinces is intended or should be inferred.

Collaborators The Canadian Network for Observational Drug Effect Studies (CNODES) Investigators are: Samy Suissa (Principal Investigator); Colin Dormuth (British Columbia); Brenda Hemmelgarn (Alberta); Gary Teare (Saskatchewan); Patricia Martens and Patricia Caetano (Manitoba); David Henry and Michael Paterson (Ontario); Jacques LeLorier (Québec); Adrian Levy (Nova Scotia); Pierre Ernst (GPRD); Robert Platt (Methods); and Ingrid Sketris (Knowledge Translation).

Contributors All authors were involved in the study conception and design, statistical analysis and interpretation of data. KBF drafted the manuscript, and all authors critically reviewed the manuscript for important intellectual content.

Funding CNODES is a collaborating center of the Drug Safety and Effectiveness Network (DSEN), funded by the Canadian Institutes of Health Research (Grant Number DSE-111845). The funding source was not involved in the writing of the manuscript or the decision to submit it for publication.

Competing interests $\mathrm{PE}$ has received speaker fees and has sat on advisory boards for AstraZeneca, Boehringer Ingelheim, GlaxoSmithKline, Merck, Novartis and Nycomed, all prior to January 2012. LET has received speakers' fees from Pfizer Canada, and has sat on advisory boards for Astra Zeneca Canada, Janssen Canada and Merck Canada.

Ethics approval Research Ethics was obtained at all eight participating sites, including the Jewish General Hospital, Montreal, Quebec.

Provenance and peer review Not commissioned; externally peer reviewed.

Open Access This is an Open Access article distributed in accordance with the Creative Commons Attribution Non Commercial (CC BY-NC 3.0) license, which permits others to distribute, remix, adapt, build upon this work non-commercially, and license their derivative works on different terms, provided the original work is properly cited and the use is non-commercial. See: http://creativecommons.org/ licenses/by-nc/3.0/

\section{REFERENCES}

1 Katz MH. Failing the acid test: benefits of proton pump inhibitors may not justify the risks for many users. Arch Intern Med 2010;170:747-8.

2 Heidelbaugh JJ, Goldberg KL, Inadomi JM. Overutilization of proton pump inhibitors: a review of cost-effectiveness and risk [corrected]. Am J Gastroenterol 2009;104(Suppl 2):S27-32

3 Eom CS, Jeon CY, Lim JW, et al. Use of acid-suppressive drugs and risk of pneumonia: a systematic review and meta-analysis. CMAJ 2011;183:310-19.

4 Gaude GS. Pulmonary manifestations of gastroesophageal reflux disease. Ann Thorac Med 2009:4:115-23.

5 el-Serag HB, Sonnenberg A. Comorbid occurrence of laryngeal or pulmonary disease with esophagitis in United States military veterans. Gastroenterology 1997;113:755-60.

6 Hermos JA, Young MM, Fonda JR, et al. Risk of community-acquired pneumonia in veteran patients to whom proton pump inhibitors were dispensed. Clin Infect Dis 2012;54:33-42.

7 Gulmez SE, Holm A, Frederiksen $\mathrm{H}$, et al. Use of proton pump inhibitors and the risk of community-acquired pneumonia: a population-based case-control study. Arch Intern Med 2007;167:950-5.

8 Abraham NS, Hlatky MA, Antman EM, et al. ACCF/ACG/AHA 2010 expert consensus document on the concomitant use of proton pump inhibitors and thienopyridines: a focused update of the ACCF/ACG/AHA 2008 expert consensus document on reducing the gastrointestinal risks of antiplatelet therapy and NSAID use. Am J Gastroenterol 2010;105:2533-49.

9 Burmester G, Lanas A, Biasucci L, et al. The appropriate use of non-steroida anti-inflammatory drugs in rheumatic disease: opinions of a multidisciplinary European expert panel. Ann Rheum Dis 2011:70:818-22. 
10 Rostom A, Moayyedi P, Hunt R. Canadian consensus guidelines on long-term nonsteroidal anti-inflammatory drug therapy and the need for gastroprotection: benefits versus risks. Aliment Pharmacol Ther 2009;29:481-96.

11 Suissa S, Henry D, Caetano P, et al. CNODES: the Canadian Network for Observational Drug Effect Studies. Open Med 2012;6:134-40.

12 Ray WA. Evaluating medication effects outside of clinical trials: new-user designs. Am J Epidemiol 2003;158:915-20.

13 Skull SA, Andrews RM, Byrnes GB, et al. ICD-10 codes are a valid tool for identification of pneumonia in hospitalized patients aged $>0 \mathrm{r}=65$ years. Epidemiol Infect 2008;136:232-40.

14 Szklo M, Nieto FJ. Epidemiology: beyond the basics. Sudbury, MA: Aspen Publishers, 1999

15 Schneeweiss S, Rassen JA, Glynn RJ, et al. High-dimensional propensity score adjustment in studies of treatment effects using health care claims data. Epidemiology 2009;20:512-22.

16 Egger M, Smith GD, Altman DG. Systematic reviews in healthcare: meta-analysis in context. 2nd edn. London: BMJ Publishing Group, 2001.

17 Higgins JP, Thompson SG. Quantifying heterogeneity in a meta-analysis. Stat Med 2002;21:1539-58.

18 Tett SE, Sketris I, Cooke C, et al. Differences in utilisation of gastroprotective drugs between 2001 and 2005 in Australia and Nova Scotia, Canada. Pharmacoepidemiol Drug Saf Published Online First: 4 Apr 2013. doi: 10.1002/pds.3442.

19 Laheij RJ, Sturkenboom MC, Hassing RJ, et al. Risk of community-acquired pneumonia and use of gastric acid-suppressive drugs. JAMA 2004;292:1955-60.

20 Sarkar M, Hennessy S, Yang YX. Proton-pump inhibitor use and the risk for community-acquired pneumonia. Ann Intern Med 2008;149:391-8.

21 Myles PR, Hubbard RB, McKeever TM, et al. Risk of community-acquired pneumonia and the use of statins, ace inhibitors and gastric acid suppressants: a population-based case-control study. Pharmacoepidemiol Drug Saf 2009;18:269-75.

22 Rodriguez LA, Ruigomez A, Wallander MA, et al. Acid-suppressive drugs and community-acquired pneumonia. Epidemiology 2009;20:800-6.
23 Roughead EE, Ramsay EN, Pratt NL, et al. Proton-pump inhibitors and the risk of antibiotic use and hospitalisation for pneumonia. Med J Aust 2009;190:114-16.

24 Johnstone J, Nerenberg K, Loeb M. Meta-analysis: proton pump inhibitor use and the risk of community-acquired pneumonia. Aliment Pharmacol Ther 2010:31:1165-77.

25 Dublin S, Walker RL, Jackson ML, et al. Use of proton pump inhibitors and $\mathrm{H} 2$ blockers and risk of pneumonia in older adults: a population-based case-control study. Pharmacoepidemiol Drug Saf 2010;19:792-802.

26 Rascon-Aguilar IE, Pamer M, Wludyka $\mathrm{P}$, et al. Role of gastroesophageal reflux symptoms in exacerbations of COPD. Chest 2006;130:1096-101.

27 Metheny NA, Chang YH, Ye JS, et al. Pepsin as a marker for pulmonary aspiration. Am J Crit Care 2002;11:150-4.

28 Jena $A B$, Sun $E$, Goldman DP. Confounding in the association of proton pump inhibitor use with risk of community-acquired pneumonia. J Gen Intern Med 2013;28:223-30.

29 Estborn L, Joelson S. Occurrence of community-acquired respiratory tract infection in patients receiving esomeprazole: retrospective analysis of adverse events in 31 clinical trials. Drug Saf 2008;31:627-36.

30 McGraw D, Rosati K, Evans B. A policy framework for public health uses of electronic health data. Pharmacoepidemiol Drug Saf 2012;21(Suppl 1):18-22.

31 Behrman RE, Benner JS, Brown JS, et al. Developing the Sentinel System-a national resource for evidence development. N Engl J Med 2011;364:498-9.

32 Roos LL, Gupta S, Soodeen RA, et al. Data quality in an information-rich environment: Canada as an example. Can J Aging 2005;24(Suppl 1):153-70.

33 Khan NF, Harrison SE, Rose PW. Validity of diagnostic coding within the General Practice Research Database: a systematic review. Br J Gen Pract 2010;60:e128-36.

34 Canadian Institute for Health Information. CIHI data quality study of the 20062007 Discharge Abstract Database. Ottawa, Canadian Institute for Health Information, 2009, 12-5-2013.

35 Lindenauer PK, Lagu T, Shieh MS, et al. Association of diagnostic coding with trends in hospitalizations and mortality of patients with pneumonia, 2003-2009. JAMA 2012;307:1405-13. 